\title{
Expression and Role of CD166 in the Chronic Kidney Disease
}

\author{
Yan Sun, ${ }^{1,2}$ Yiping Wang, ${ }^{2}$ Qi Cao, ${ }^{2}$ Hong Yu, Dong Zheng, ${ }^{2}$ Ya Wang, ${ }^{2}$ and David C. H. Harris ${ }^{2}$ \\ ${ }_{1}^{1}$ Department of Pediatrics, Shandong Provincial Hospital Affiliated to Shandong University, Jinan, China \\ ${ }^{2}$ Centre for Transplantation and Renal Research, University of Sydney at Westmead Millennium Institute, Sydney, Australia \\ ${ }^{3}$ The High Tech Centre, Westmead Millennium Institute, University of Sydney Westmead, Sydney, Australia \\ *Corresponding author: Yan Sun, Department of Pediatrics, Shandong Provincial Hospital Affiliated to Shandong University, Jinan, China. Tel:+86-15168889589, E-mail: sunyan6150@126.com \\ Received: February 5, 2015; Accepted: February 16, 2015
}

\begin{abstract}
Background: CD166, an adhesion molecule of the immunoglobulin superfamily, is one of the crucial effectors that traffic lymphocytes into tissues. Till now, the expression and role of CD166 in the chronic kidney disease remains unknown.

Objectives: In the present study, we are to examine the expression of CD166 in the chronic kidney disease, and to explore its function with CD4+T cells.

Materials and Methods: CD166 expression was tested by Flow Cytometry (FACS) in the primary macrophages stimulated with LPS. In vivo, the expression of CD166 and CD4 were examined in the kidney tissues of adriamycin-induced nephropathy (AN) mice by immnohistochemistry. Macrophages and lymphocytes were co-cultured, the interaction between CD166 and CD4 was tested by immunofluorescent staining. Furthermore, the effects of CD166 on the activation and proliferation of T cells were explored.

Results: In this study, CD166 expression was found to be upregulated on activated macrophages and glomerular endothelia in the adriamycin-induced nephropathy (AN) mice and CD4+ T cells were increased with CD166 expression in the AN mice. The interaction between macrophages and CD4+ T cells indicated that CD166 played a key role in the recruitment of lymphocytes in the chronic kidney disease, and neither proliferation nor activation of T cells was affected by CD166.

Conclusions: CD166 expressed on macrophages and endothelia in AN kidney, and the function was related to the recruitment of CD4+ T cells into inflamed kidney, indicating that CD166 may be a potential target for reducing the inflammatory infiltrates in the chronic kidney disease.
\end{abstract}

Keywords: Activated-Leukocyte Cell Adhesion Molecule; Lymphocytes; Chronic Kidney Disease

\section{Background}

CD166, also called activated leukocyte cell adhesion molecule (ALCAM), is initially found as a ligand for CD6, the cell surface receptor of T lymphocyte $(1,2)$. CD6 involves in the activation and differentiation of $\mathrm{T}$ cells. A rat $\mathrm{mAb}$ specific for mCD166 was produced and shown to bind to both activated CD4+ and CD8+ T cells by twocolor immunofluorescence studies. The structure-function analyses of CD166 hint its cytoskeletal anchoring and the integrity of the extracellular immunoglobulinlike domains may regulate complex cellular properties with regard to cell adhesion, growth and migration (3). Expressed on activated monocytes and endothelial cells, CD166 has been reported to be involved in various cell migration processes such as neurogenesis, blastocyst implantation, neurite outgrowth, melanoma invasion and tumor progression (4-7). The localization of CD166 specifically at cell-cell junctions on endothelial cells supports its role in transendothelial migration (8). CD166 has also been reported to be expressed in blood-brain-barrier (BBB), and CD166 blockade has restricted the transmigration of CD4+ lymphocytes and monocytes across endothelium. The findings indicate an important role of CD166 in the recruitment of CD4+ T cells.

As known, the infiltration of leukocytes and chemokines is the feature in most types of renal inflammation (9-11). mRNA transcripts encoding CD166 have been found to be expressed in kidney tissue. It has been recently reported that the von Hippel-Lindau (VHL) tumor suppressor gene plays a central role in development of clear cell renal cell carcinoma. The VHL targets include upregulated CD166, which may be important in tumorigenesis (12). The mesenchymal stem cells in renal disease were over $90 \%$ positive for $\mathrm{CD} 166$, and negative for $\mathrm{CD} 31$. In response to immune activation they both increased the expression of pro-inflammatory and anti-inflammatory factors (13). While the expression and role of CD166 in the chronic kidney disease remains unknown.

Copyright (C) 2015, Growth \& Development Research Center. This is an open-access article distributed under the terms of the Creative Commons Attribution-NonCommercial 4.0 International License (http://creativecommons.org/licenses/by-nc/4.0/) which permits copy and redistribute the material just in noncommercial usages, provided the original work is properly cited. 
Sun Yetal.

\section{Objectives}

In the present study, we examined the expression of CD166 in the chronic kidney disease, and explored its function with CD4+ T cells.

\section{Materials and Methods}

\subsection{Mice and Adriamycin-Induced Nephropathy Models}

Female BALB/c mice (4 - 6 w, Center of Animal Research, Westmead Hospital, Australia) were used for this study. All the experiments were approved by Australian Guide for the Care and Use of Laboratory Animals.

Murine models of adriamycin-induced nephropathy (AN) were established by adriamycin injection $(10.2 \mathrm{mg} /$ $\mathrm{kg}, \mathrm{n}=8$, Pharmacia and Upjohn Pty Ltd. Australia) via tail vein. Four weeks after injection, AN mice and normal control mice $(n=8)$ were sacrificed, and kidney tissues were harvested and dealt as described previously (14).

\subsection{Cell Culture}

Primary macrophages were isolated from the spleens of normal mice by being crushed on the $40 \mu \mathrm{m}$ filter in the plate filled with RPMI. Then, splenocytes were isolated and cultured overnight by the technique previously described (15).

CD4+ T cells were isolated using microbeads. Splenocytes were purified with anti-biotin $\left(200 \mu \mathrm{L} / 10^{8}\right.$ cells $) \mathrm{Mi}$ croBeads. After washing, cells were resuspended in $500 \mu \mathrm{L}$ MACS buffer and added into the columns. The cells in the effluent are CD4+ T cells. Macrophages were grown for 48 hours in the 24 -well plate. Then, $10^{4}$ isolated CD4+ T cells $\left(100 \mu \mathrm{L}, 10^{6}\right.$ cells $\left./ \mathrm{mL}\right)$ were added into every well to coculture for 6 hours. And the localization of macrophage and CD4+ T cell was examined by live cell microscope (Leica).

\subsection{Immunostaining}

For immunocytochemistry, CD166 and CD4 expression in kidney tissues were detected with antibodies (CD166 antibody, Goat anti mouse, 1:30,R and D system; CD4 antibody, Rat anti mouse, 1:50, BD Pharmingen, USA) as usual (16); and the percentages of positive cells were examined. Immunofluorescent staining was conducted as follows. Kidney slides were double stained with the mixture of both primary antibodies against CD166 and CD4, then cultured with the mixture of two fluorescent secondary antibodies (FITC-conjugated donkey anti goat antibody, 1:100, Santa Cruz, USA; Rhodamine-conjugated rabbit anti rat antibody, 1:200, Rockland, USA). The slides were rinsed with PBS, and mounted with Dako cytomation. All images were visualized on a Leica SP5 confocal microscope (Leica TCS SP5) and were analyzed with Leica LAS AF software (Leica Wetzlar).

\subsection{Flow Cytometry (FACS)}

Primary macrophages were treated with LPS $(1 \mathrm{~g} / \mathrm{mL})$ for 20 hours. Then, cells with or without treatment were collected in cold PBS and were incubated with monoclonal PE-conjugated CD166 antibody (eBioscience). FACS analysis was performed on a FACSCALIBUR (BD Biosciences, San Jose, CA) as previously described (17). Data was analyzed with FACSDiva software.

\subsection{TCell Activation}

The following four groups were studied: a, control cells + IgG1; b, control cells + CD166 antibody; c, LPS-stimulated cells + IgG1; d, LPS-stimulated cells + CD166 antibody.

Macrophages were grown for 48 hours, and cells in group c and d were added with LPS $(1 \mu \mathrm{g} / \mathrm{mL})$ for 8 hours. CD4+ T cells and macrophages were co-cultured for 18 hours. Antibodies specific for CD166 or the isotype IgG1 were added 3 hours before starting co-culture. Then T cells were collect and followed by RNA isolation, RT-PCR and Real-time RT-PCR was performed as previously described (18). RNA was converted to cDNA using SuperScript III Reverse Transcriptase and oligo (dT) 20 (Invitrogen Life Technologies). The activation of T cells was judged by levels of IFN- $\gamma$ (19). IFN- $\gamma$ level was normalized to that of $\beta$-Actin. The primers were as follows: $\beta$-Actin, 5'-CAAGTCATCA CTATTGGCAACG A-3' and 5'-CCCAAGAAGGAAGGCTGGA-3'; IFN- $\gamma$, 5'-CAGCAACAGCAAGGCGAA-3' and 5'-CTGGACCTGTGGGTTGTTGAC-3'.

\subsection{Quantification of T Cell Proliferation (WST-1)}

Here also four groups were studied. LPS-stimulation and CD166 blockade were performed as above. Macrophags and CD4+ T cells were co-cultured for 3 days in 96well microplates. Then, cell proliferation reagent WST-1 (10 L/well) was added. After 4 hours incubation, the absorbance representing the proliferation of $\mathrm{T}$ cells was measured using a microplate (ELISA) reader at $450 \mathrm{~nm}$.

\subsection{PAS Staining}

In PAS staining, paraffin slides were de-paraffinized, rehydrated and ended in water. The slides were placed in 2\% Periodic Acid for 15 minutes and then, stained with Schiff's reagent for 25 minutes. Counter-staining with filtered Hematoxylin was conducted. After three washes, the slides were dipped quickly in acid alcohol for two and slowly in ammonia for four times. Finally, the slides were dehydrated and cover slipped.

\subsection{Statistical Analyses}

SPSS software (11.0) was used for statistical analyses, which included analysis of variance followed by ANOVA. Only P values of less than 0.05 were considered statistically significant. 


\section{Results}

\subsection{Increased CD166 Expression on Macrophages by LPS Stimulation}

Macrophages were derived from BALB/c mice. CD166 expression of macrophages was only detectable on untreated macrophages, but increased significantly after LPS stimulation (1.64\% vs. 7.78\%, $\mathrm{P}<0.05, \mathrm{n}=3$; Figure 1 ).

\subsection{Increased CD166 and CD4 Expression in $A N$} Kidney Tissues

In AN mice with PAS staining, kidneys showed glomerular sclerosis, tubule atrophy and interstitial expansion. Also, large amounts of inflammatory infiltration appeared in interstitium (Figure 2).

Figure 1. CD166 Expression Increased on the LPS-Stimulated Macrophage

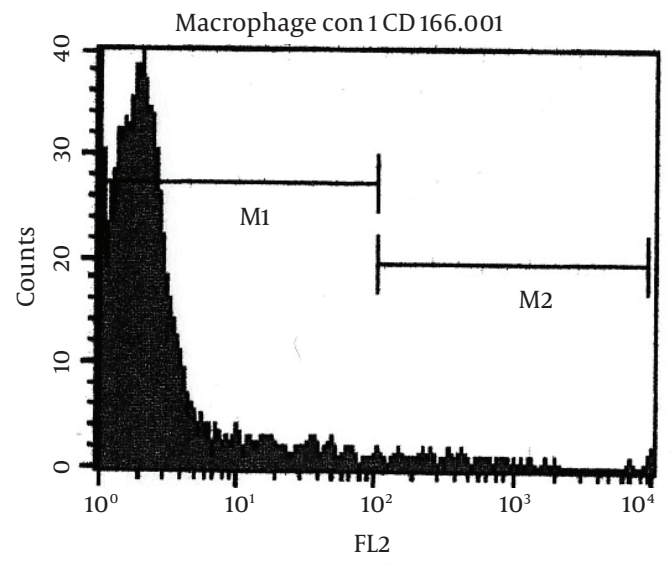

Histogram Statistics

\begin{tabular}{cc} 
Marker \%Gated \\
\hline All & 100.00 \\
M1 & 98.33 \\
M2 & 1.64
\end{tabular}

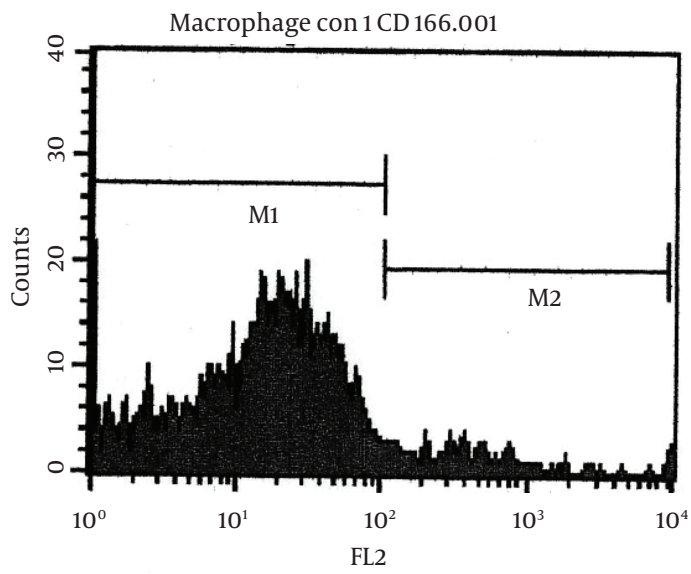

Histogram Statistics

\begin{tabular}{cc} 
Marker \%Gated \\
\hline All & 100.00 \\
M1 & 92.12 \\
M2 & 7.85
\end{tabular}

CD166 expression examined by FACS, was increased significantly from 1.64\% to 7.78\% after LPS stimulation (P<0.05; $\mathrm{n}=3$ ). A, primary macrophage, B, LPSstimulated primary macrophage.

Figure 2. Kidney Injury of AN Mice in PAS Staining
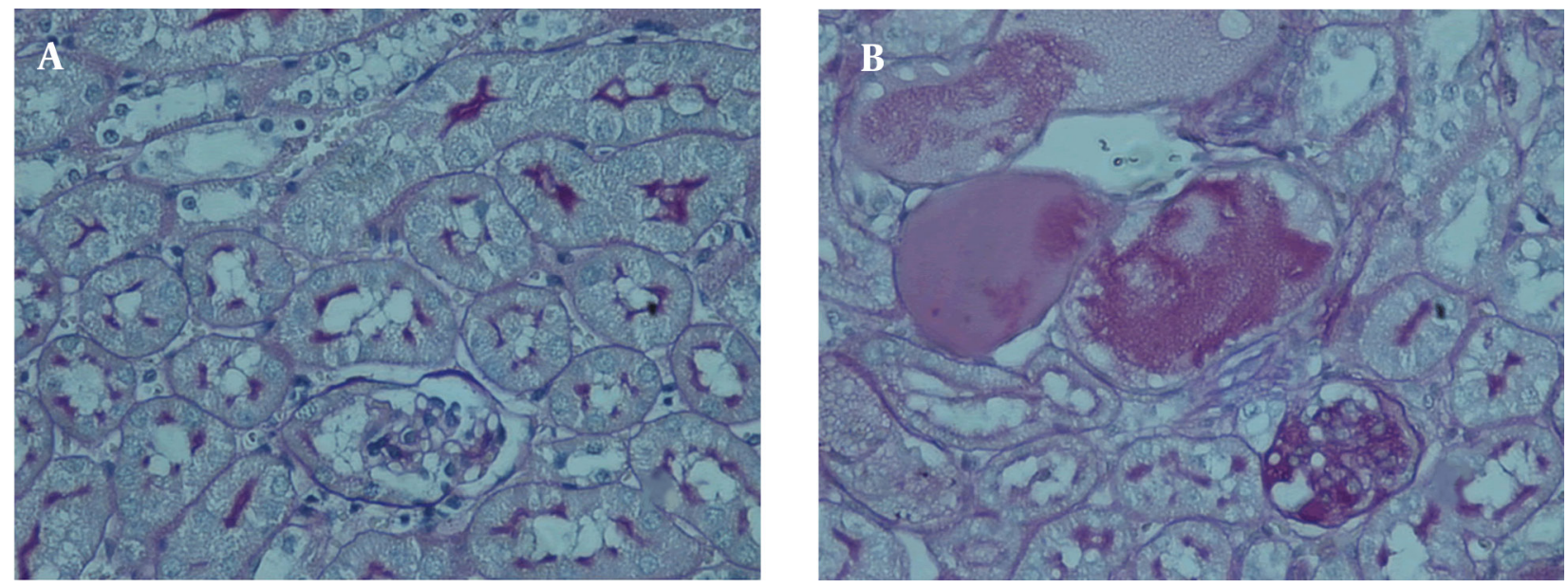

In PAS staining, AN mice developed expanded interstitial area, severe glumerular sclerosis and reduced tubular height by PAS staining, $(\times 400)$. 

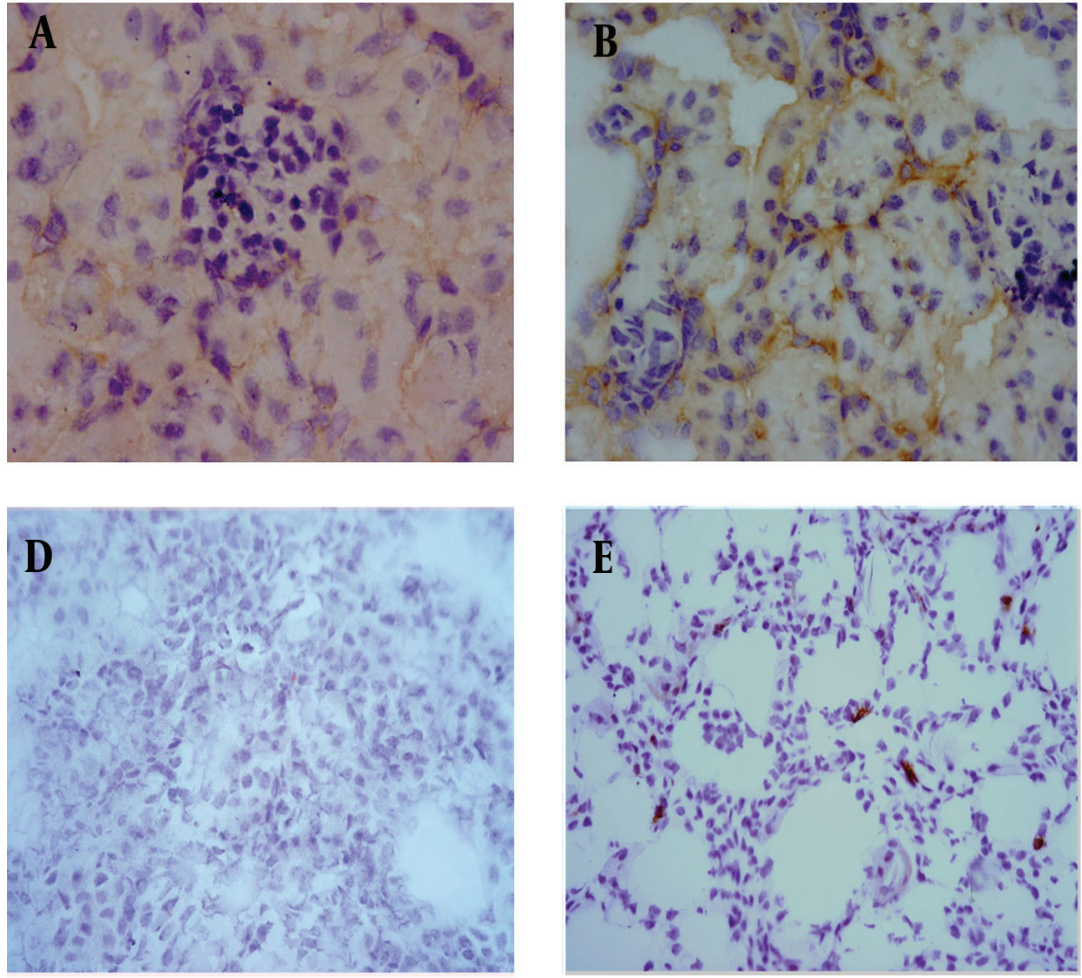

C
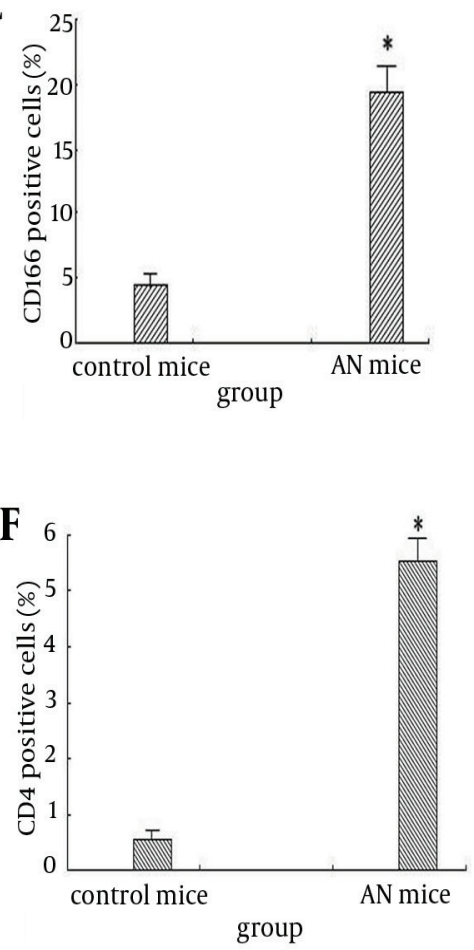

CD166, weakly expressed in the normal mice, was expressed much greater in glomeruli and infiltrated macrophages in AN kidney tissues. CD4 was expressed mostly in the interstitial area of kidney tissues, increased in AN mice compared with that in the normal mice. A, CD166 expression in normal mice, $\times 400$; B, CD166 expression in the AN mice, $\times 400$; C, CD166 positive cells (\%); D, CD4 expression in the normal mice, $\times 200$; E, CD4 expression in the AN mice, $\times 200$. $F$, CD4 positive cells $(\%)$.

We assessed the expression of CD166 and CD4 in kidney tissues of AN mice by immunohistochemistry (Figure 3). CD166 was expressed weakly in the normal kidney, but greatly increased in glomeruli and infiltrated macrophages in the interstitial area of kidney tissues (4.40\% vs. $19.42 \%, \mathrm{P}<0.05)$. CD $4+\mathrm{T}$ cells were mostly in the interstitium of kidney, and increased in AN mice compared with the controls ( $0.56 \%$ vs. $5.53 \%, \mathrm{P}<0.05)$.

\subsection{CD166 Positive Cells Interact With Lymphocytes}

CD166 positive cells were found to connect with CD4+ T cells closely by double staining in the AN kidney tissues (Figure 4). Similar results were shown in the co-culture of primary macrophages and CD4+ T cells (Figure 5), suggesting that CD166 could attract the migration of lym- phocytes by interaction with its effector. Thus CD166 attracts recruitment of CD4+ T cells into the kidney tissues in chronic kidney disease.

\subsection{Effects of CD166 on T Cell Activation and Pro- liferation}

The IFN- $\gamma$ levels in T cells co-cultured with macrophages were significantly increased after LPS stimulation (A, P < $0.05 ; n=3$. Figure 6 ), while there was no significant difference after CD166 antibody was added. Similarly, T cell proliferation was increased after LPS stimulation, but there was no significant difference after given CD166 antibody $(\mathrm{B}, \mathrm{P}<0.05 ; \mathrm{n}=3)$. Thus, $\mathrm{CD} 166$ had no obvious effects on $\mathrm{T}$ cell activation and proliferation. 
Figure 4. CD166 and CD4 Expression in AN Kidney by Immunofluorescence Staining
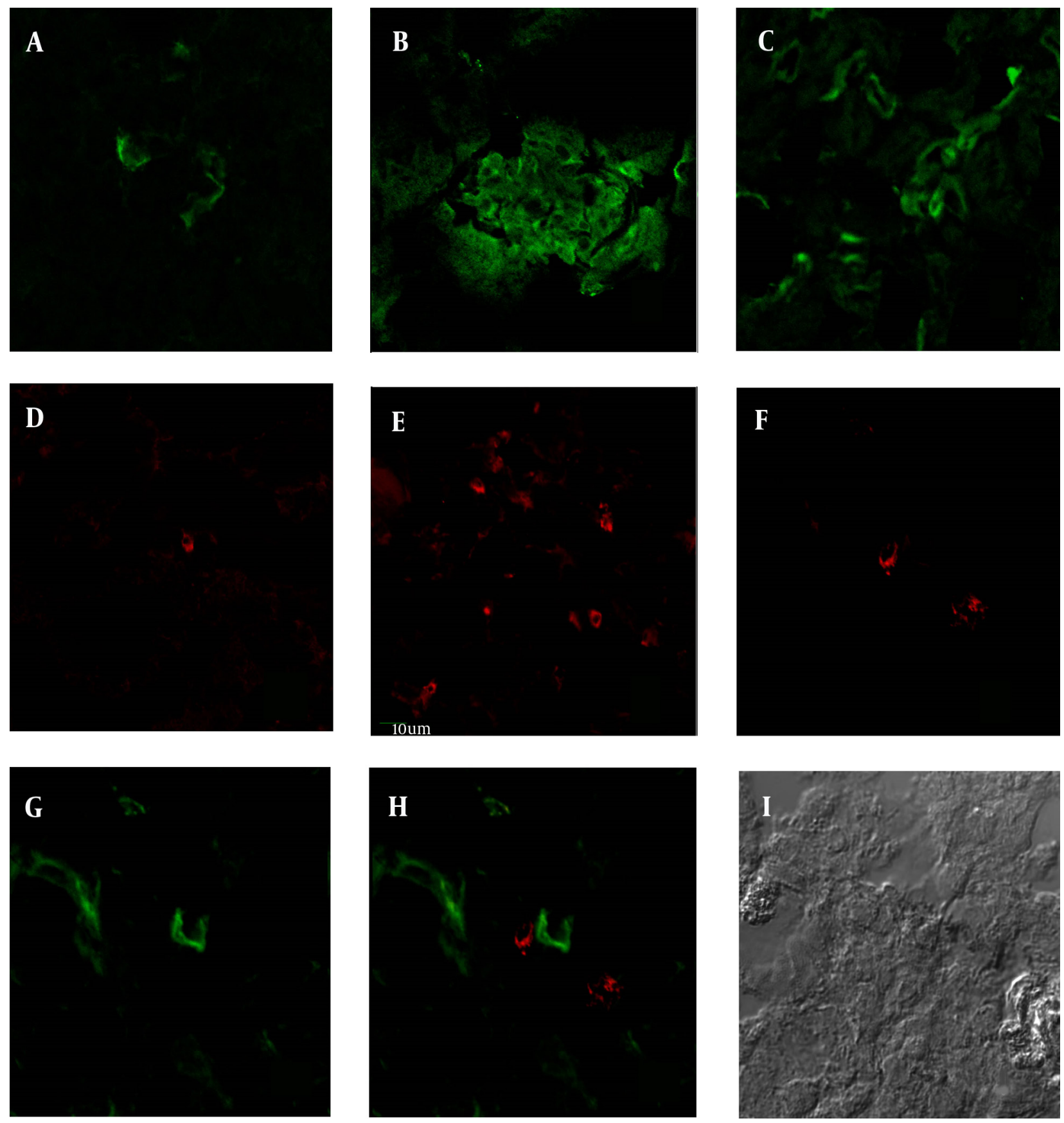

In immunofluorescence staining, CD166 and CD4 staining were significantly stronger in AN mice than in normal mice, similar to the results in the immunohistochemistry. A, CD166 expression in the normal mice; B, CD166 expression in the glomeruli of AN mice; C, CD166 expression in the interstitial area of AN mice; D, CD4 expression in the normal mice; E, CD4 expression in the AN mice; F, CD4 expression in the double staining in AN kidney (Rodamine channel); G, CD166 expression in the double staining in AN kidney (FITC channel); H, double staining of CD166 and CD4 in AN kidney (CD4-Rodamine channel, CD166-FITC channel); I, DIC image of double staining $(\times 400)$. 
Figure 5. The Interaction Between Macrophage and CD4+ T Cells Under Live Cell Microscope
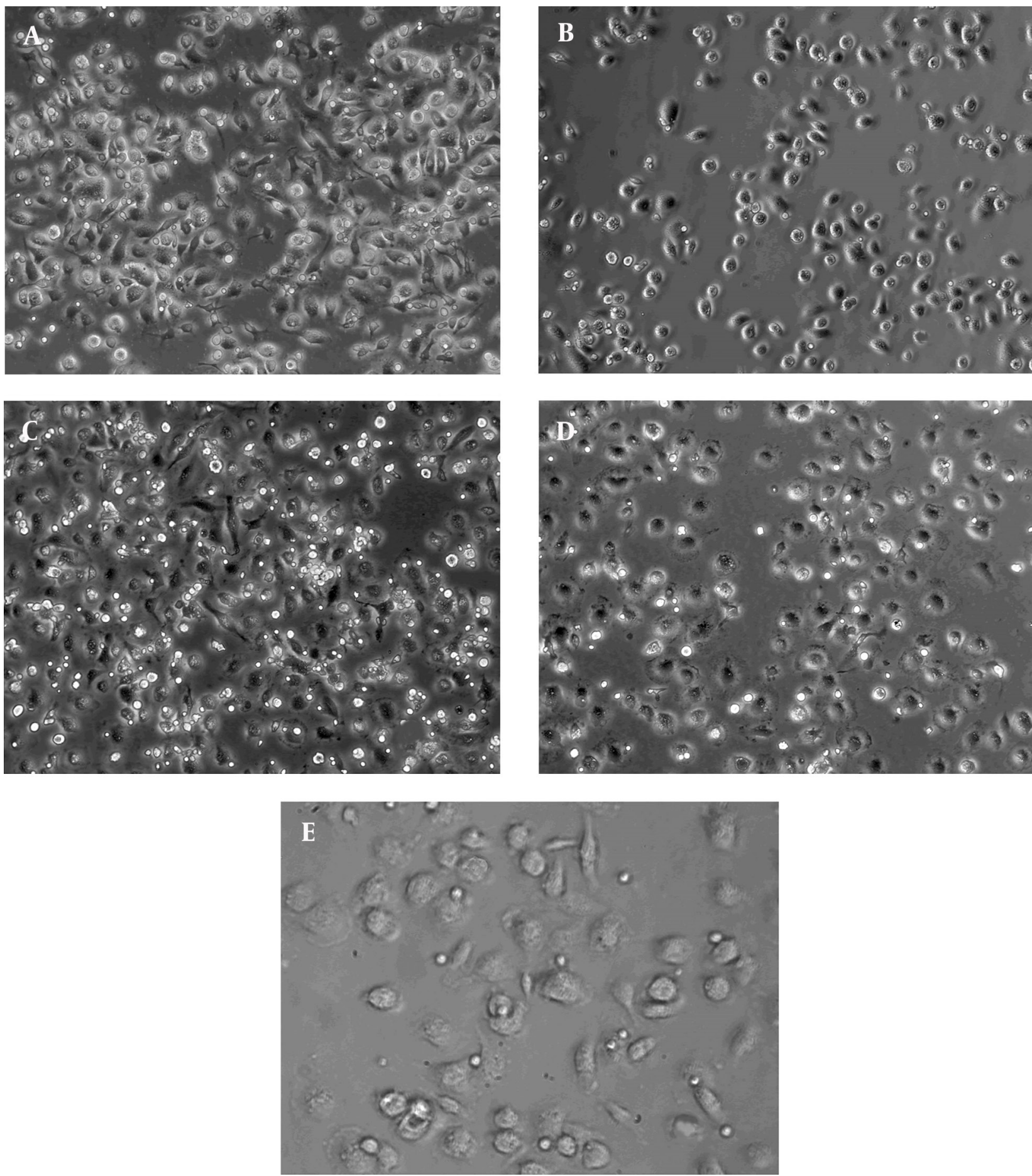

The macrophages and CD4+ T cells in the chamber were visualized using the live cell macroscope. The images of the localization of CD4+ T cells and CD166 + macrophages, showed the accumulation of T lymphocytes around macrophages. A, control + IgG1 group; B, control + CD166-Ab group; C, LPS + IgG1 group; D, LPS + CD166-Ab group; E, interaction of macrophages and CD4+ T cells; $(\times 200)$ 
A

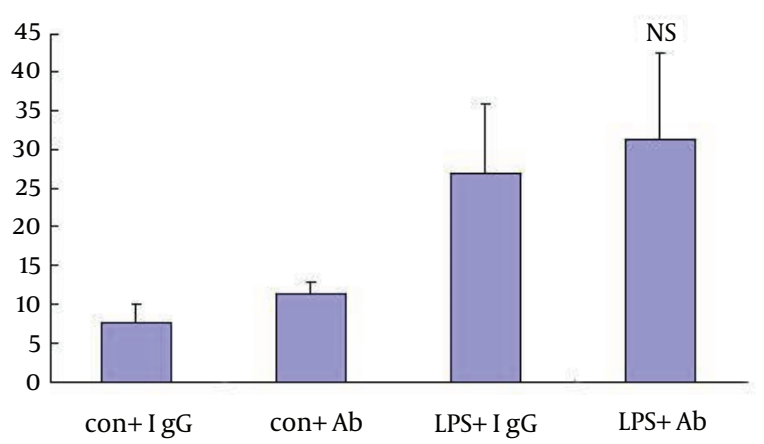

B

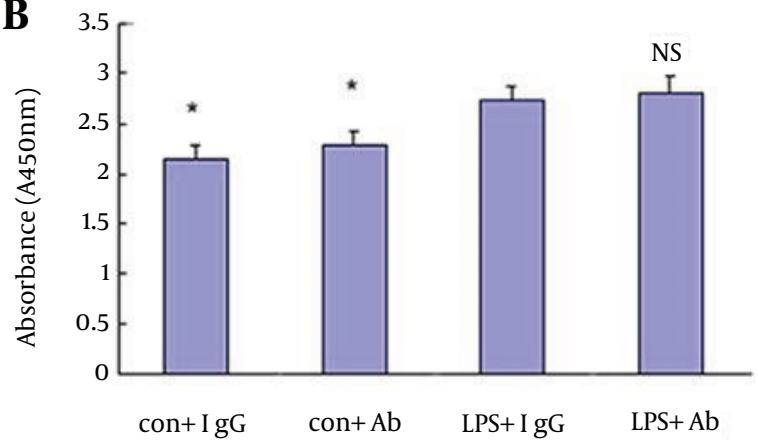

The IFN- $\gamma$ level in T cells co-cultured with macrophage was significantly increased after LPS stimulation $(\mathrm{A}, \mathrm{P}<0.05, \mathrm{n}=3)$, while no difference was shown between the CD166 antibody group and the IgG1 group. In the proliferation experiment, the absorbance representative of T cell proliferation was increased after LPS stimulation, while there was no significant difference in the effects on T cell proliferation between CD166 antibody and isotype IgG1 (B, $\mathrm{P}<0.05, \mathrm{n}=3)$.

\section{Discussion}

The study has shown that CD166 expression was up-regulated in AN, expressed strongly on infiltrated macrophages and glomerular endothelia. In vitro, CD166 expression on macrophages was also increased after LPS stimulation, which indicated that CD166 might be a hallmark of activated macrophages. CD166 is the crucial ligand for T lymphocyte, CD4 expression was also increased mostly in the kidney tissues of AN mice. And their closely interaction indicated that CD166 was important in the recruitment of lymphocytes in the chronic kidney disease.

CD166 expression was consistently up-regulated in both LPS-stimulated macrophages and kidney tissues of AN mice. CD4+ T lymphocytes were also increased in the AN mice. As known, effectiveness of the adaptive immune system is largely dependent on its ability to deliver specific $\mathrm{T}$ cell subsets to requisite sites of inflammation. In the chronic kidney disease, podocyte loss in the glomeruli was identified as the central event in the development of glomerulosclerosis. The trigger is dysfunction or absence of podocytes that stabilize the slit diaphragm or anchor foot processes to the basement membrane. Sustained injury of the glomerular barrier to protein is transmitted to the tubulointerstitial compartment leading to inflammation and fibrosis (20). Chronic kidney disease describes a form of intrinsic kidney injury that results from persistent activation of the immune system. Macrophages play an important role in the induction of anti-glomerular basement membrane glomerulonephritis and glomerular injury (21) and relative lymphocyte counts as a marker of progression of chronic kidney disease (22). It has been proven that CD166 expression was consistently up-regulated in both LPS-stimulated macrophages and kidney tissues of AN mice, and CD4+ T lymphocytes were also increased in the AN mice. Thus increased macrophages and $\mathrm{T}$ cells infiltrate into the interstitial areas, and inflammation process starts to impair the glomerular barrier in chronic kidney disease.

Accumulation of specific effector cells in inflammatory lesions such as kidney tissues derives from dynamic processes orchestrated in part by expression of chemokine receptors. CD166 is a member of the immunoglobulin superfamily of cell adhesion molecules which mediate intercellular adhesion through either homophilic (CD166CD166) or heterophilic (CD166-CD6) interactions (23). CD166-mediated adhesion is crucial in different physiological and pathological phenomena, with particular relevance in leukocyte extravasation, and stabilization of the immunological synapses (24). In our study, the expression of both CD166 and CD4 were increased in the glomerular endothelia and the interstitial areas in AN mice. The interaction between macrophages and CD4+ T cells, indicates that CD166 is important in the T-cell trafficking towards macrophages and glomerular endothelia in the chronic kidney disease. The functional role of CD166 is, as it was suggested, dependent in part by interaction of CD166 and its ligand pathways. Furthermore, the function of CD166 in the development of chronic kidney disease is related to its ability to mediate CD4+ T cells migration, rather than its effect on the activation and proliferation of $\mathrm{T}$ cells.

CD166 blockade has been reported to restrict the transmigration of CD4+ lymphocytes across BBB endothelium 
and reduce the severity of experimental autoimmune encephalomyelitis. And in this way, CD166 may be considered as a potential target for reducing the inflammatory infiltrates in the chronic kidney disease.

\section{Acknowledgements}

Thanks for the funding from China Scholarship Council. Thanks for the research guidence and supports by Yiping Wang. And thanks for the life support for the first author from China Scholarship Council.

\section{References}

1. Gimferrer I, Calvo M, Mittelbrunn M, Farnos M, Sarrias MR, Enrich C, et al. Relevance of CD6-mediated interactions in T cell activation and proliferation. JImmunol. 2004;173(4):2262-70.

2. Ibanez A, Sarrias MR, Farnos M, Gimferrer I, Serra-Pages C, Vives J, et al. Mitogen-activated protein kinase pathway activation by the CD6 lymphocyte surface receptor. JImmunol. 2006;177(2):1152-9.

3. Swart GW. Activated leukocyte cell adhesion molecule (CD166/AL CAM): developmental and mechanistic aspects of cell clustering and cell migration. Eur J Cell Biol. 2002;81(6):313-21.

4. Ohneda O, Ohneda K, Arai F, Lee J, Miyamoto T, Fukushima Y, et al. ALCAM (CD166): its role in hematopoietic and endothelial development. Blood. 2001;98(7):2134-42.

5. Jezierska A, Matysiak W, Motyl T. ALCAM/CD166 protects breast cancer cells against apoptosis and autophagy. Med Sci Monit 2006;12(8):BR263-73.

6. Ofori-Acquah SF, King JA. Activated leukocyte cell adhesion molecule: a new paradox in cancer. Transl Res. 2008;151(3):122-8.

7. van Kempen LC, van den Oord JJ, van Muijen GN, Weidle UH, Bloemers HP, Swart GW. Activated leukocyte cell adhesion molecule/CD166, a marker of tumor progression in primary malignant melanoma of the skin. Am JPathol. 2000;156(3):769-74.

8. Masedunskas A, King JA, Tan F, Cochran R, Stevens T, Sviridov D, et al. Activated leukocyte cell adhesion molecule is a component of the endothelial junction involved in transendothelial monocyte migration. FEBS Lett. 2006;580(11):2637-45.

9. Ikezumi Y, Kanno K, Karasawa T, Han GD, Ito Y, Koike H, et al. The role of lymphocytes in the experimental progressive glomerulonephritis. Kidney Int. 2004;66(3):1036-48.

10. Segerer S, Djafarzadeh R, Grone HJ, Weingart C, Kerjaschki D Weber C, et al. Selective binding and presentation of CCL5 by discrete tissue microenvironments during renal inflammation. Am Soc Nephrol. 2007;18(6):1835-44.
11. Ruster C, Wolf G. The role of chemokines and chemokine receptors in diabetic nephropathy. Front Biosci. 2008;13:944-55.

12. Aggelis V, Craven RA, Peng J, Harnden P, Cairns DA, Maher ER, et al. Proteomic identification of differentially expressed plasma membrane proteins in renal cell carcinoma by stable isotope labelling of a von Hippel-Lindau transfectant cell line model. Proteomics. 2009;9(8):2118-30.

13. Roemeling-van Rhijn M, Reinders ME, de Klein A, Douben $\mathrm{H}$, Korevaar SS, Mensah FK, et al. Mesenchymal stem cells derived from adipose tissue are not affected by renal disease. Kidney Int. 2012;82(7):748-58.

14. Cao Q, Wang Y, Zheng D, Sun Y, Wang Y, Lee VW, et al. IL-10/TGFbeta-modified macrophages induce regulatory T cells and protect against adriamycin nephrosis. J Am Soc Nephrol. 2010;21(6):933-42.

15. Cao Q, Wang Y, Zheng D, Sun Y, Wang C, Wang XM, et al. Failed renoprotection by alternatively activated bone marrow macrophages is due to a proliferation-dependent phenotype switch in vivo. Kidney Int. 2014;85(4):794-806.

16. Chi V, Chandy KG. Immunohistochemistry: paraffin sections using the Vectastain ABC kit from vector labs.J Vis Exp. 2007;(8):308.

17. Egwuagu CE, Yu CR, Zhang M, Mahdi RM, Kim SJ, Gery I. Suppressors of cytokine signaling proteins are differentially expressed in Th1 and Th2 cells: implications for Th cell lineage commitment and maintenance. J Immunol. 2002;168(7):3181-7.

18. Egwuagu CE, Sztein J, Mahdi RM, Li W, Chao-Chan C, Smith JA, et al. IFN-gamma increases the severity and accelerates the onset of experimental autoimmune uveitis in transgenic rats. J Immunol. 1999;162(1):510-7.

19. Suga T, Mitani A, Mogi M, Kikuchi T, Fujimura T, Takeda H, et al Aggregatibacter actinomycetemcomitans lipopolysaccharide stimulated epithelial cells produce interleukin-15 that regulates T cell activation. Arch Oral Biol. 2013;58(10):1541-8.

20. Zoja C, Abbate M, Remuzzi G. Progression of chronic kidney disease: insights from animal models. Curr Opin Nephrol Hypertens. 2006;15(3):250-7.

21. Isome M, Fujinaka H, Adhikary LP, Kovalenko P, El-Shemi AG, Yoshida $Y$, et al. Important role for macrophages in induction of crescentic anti-GBM glomerulonephritis in WKY rats. Nephrol Dial Transplant. 2004;19(12):2997-3004.

22. Kim SM, Kim HW. Relative lymphocyte count as a marker of progression of chronic kidney disease. Int Urol Nephrol. 2014;46(7):1395-401.

23. van Kempen LC, Nelissen JM, Degen WG, Torensma R, Weidle UH, Bloemers HP, et al. Molecular basis for the homophilic activated leukocyte cell adhesion molecule (ALCAM)-ALCAM interaction.J Biol Chem. 2001;276(28):25783-90.

24. Gilsanz A, Sanchez-Martin L, Gutierrez-Lopez MD, Ovalle S, Machado-Pineda Y, Reyes R, et al. ALCAM/CD166 adhesive function is regulated by the tetraspanin CD9. Cell Mol Life Sci. 2013;70(3):475-93. 